\title{
Comparative Effectiveness of Direct Admission \& Admission through Emergency Departments for Children: A Randomized Stepped Wedge Study Protocol
}

JoAnna K. Leyenaar ( $\sim$ JoAnna.K.Leyenaar@hitchcock.org )

Dartmouth-Hitchcock Medical Center https://orcid.org/0000-0002-0555-0154

Corrie E McDaniel

Seattle Children's Hospital

Stephanie Acquilano

Dartmouth College Geisel School of Medicine

Andrew Schaefer

Dartmouth College Geisel School of Medicine

Martha L. Bruce

Dartmouth-Hitchcock Medical Center

\section{A. James O'Malley}

Dartmouth College Geisel School of Medicine

\section{Study protocol}

Keywords: Implementation, Direct admission, Cluster randomized controlled trial, Pediatric hospitalizations, Multi-stakeholder teams, Pediatric hospital medicine

Posted Date: August 6th, 2020

DOI: https://doi.org/10.21203/rs.3.rs-52189/v1

License: (c) (i) This work is licensed under a Creative Commons Attribution 4.0 International License. Read Full License 


\title{
Comparative Effectiveness of Direct Admission \& Admission through Emergency Departments for Children: a randomized stepped wedge study protocol
}

\author{
JoAnna K Leyenaar MD, PhD, MPH, ${ }^{1,2}$ Corrie E McDaniel DO, ${ }^{3}$ Stephanie Acquilano MA, ${ }^{2}$ \\ Andrew Schaefer PhD, ${ }^{2}$ Martha L. Bruce PhD, MPH ${ }^{2,4}$ A. James O'Malley $\mathrm{PhD}^{2,5}$ \\ ${ }^{1}$ Department of Pediatrics, Children's Hospital at Dartmouth-Hitchcock Medical Center, \\ Lebanon, New Hampshire \\ ${ }^{2}$ The Dartmouth Institute for Health Policy \& Clinical Practice, Geisel School of Medicine at \\ Dartmouth College, Hanover, New Hampshire \\ ${ }^{3}$ Department of Pediatrics, University of Washington, Seattle Children's Hospital, Seattle, \\ Washington \\ ${ }^{4}$ Department of Psychiatry, Dartmouth-Hitchcock Medical Center, Lebanon, New Hampshire \\ ${ }^{5}$ Department of Biomedical Data Science, Geisel School of Medicine at Dartmouth College, \\ Hanover, New Hampshire
}

Corresponding Author: JoAnna K Leyenaar, JoAnna.K.Leyenaar@ hitchcock.org.

Key Words: Implementation, Direct admission, Cluster randomized controlled trial, Pediatric hospitalizations, Multi-stakeholder teams, Pediatric hospital medicine

Abbreviations: DALT, Direct Admissions Leadership Team; ED, Emergency Department; EHR, Electronic health record; HTE, heterogeneity of treatment effects; ICU, Intensive care unit; PCP, Primary care practice; PHM, Pediatric Hospital Medicine; RCT, randomized controlled trial; SSTI, skin and soft tissue infections; UTI, urinary tract infections 


\begin{abstract}
. (word count 349)
Background: Approximately 2 million children are hospitalized each year in the United States, with more than three-quarters of non-elective hospitalizations admitted through emergency departments (EDs). Direct admission, defined as admission to hospital without first receiving care in the hospital's ED, may offer benefits for patients and healthcare systems in quality, timeliness, and experience of care. While ED utilization patterns are well studied, there is a paucity of research comparing the effectiveness of direct and ED admissions. The overall aim of this project is to compare the effectiveness of a standardized direct admission approach to admission beginning in the ED for hospitalized children.
\end{abstract}

Methods/Design: We will conduct a stepped wedge cluster randomized controlled trial at 3 structurally and geographically diverse hospitals. A total of 70 primary and urgent care practice sites in the hospitals' catchment areas will be randomized to a time point when they will begin participation in the multi-stakeholder informed direct admission program. This crossover will be unidirectional and occur at 4 time points, six months apart, over a 24-month implementation period. Our primary outcome will be the timeliness of clinical care provision. Secondary outcomes include: (i) parent-reported experience of care; (ii) unanticipated transfer to the intensive care unit within 6 hours of hospital admission; and (iii) rapid response calls within 6 hours of hospital admission. We anticipate that 190 children and adolescents will be directly admitted, with 1506 admitted through EDs.

Analyses will compare the effectiveness of direct admission to admission through the ED, and will evaluate the causal effect of implementing a direct admission program using linear regression with random effects for referring practice clusters and time period fixed effects. We will further examine heterogeneity of treatment effects based on hypotheses specified a priori. In addition, we will conduct a mixed-methods process evaluation to assess reach, effectiveness, adoption, implementation and maintenance of our direct admission intervention.

Discussion: Our study represents the first randomized controlled trial to compare the effectiveness of direct admission to admission through the ED for pediatric patients. Our scientific approach, pairing a stepped-wedge design with a multi-level assessment of barriers to and facilitators of implementation, will generate valuable data about how positive findings can be reproduced across other healthcare systems.

Trial registration: This trial is registered in ClinicalTrials.gov (NCT04192799, registered December 10, 2019, https://clinicaltrials.gov/ct2/show/NCT04192799) 


\section{Contributions to the literature}

- This study represents the first randomized controlled trial to compare the effectiveness of direct admission to emergency department admission.

- By examining multi-stakeholder informed admission processes and outcomes across diverse settings, this study will expand our understanding of the factors that support and hinder hospital admission processes.

- The multi-level assessment of barriers to and facilitators of study implementation will generate valuable data about how positive findings can be reproduced in other healthcare systems. 


\section{Background}

2 Approximately two million children are admitted to hospitals in the United States (US) each

3 year. $^{1}$ These hospitalizations are costly, incurring healthcare costs that represent $40 \%$ of all

4 national pediatric healthcare expenditures. ${ }^{2,3}$ In addition, hospital admissions have major

5 implications for patients' and families' quality of life, disrupting normal routines, resulting in

6 time lost from work and school, and contributing to both financial and emotional stress. ${ }^{4}$ These

7 stressors are disproportionately experienced by children with chronic illnesses and their families,

8 who account for more than $50 \%$ of pediatric hospital admissions in the US. ${ }^{1}$

10 Over the last decade, national programs have dedicated significant resources to improving

11 hospital discharge processes: the Agency for Healthcare Research and Quality supported a major

12 initiative to re-engineer discharge practices, the Centers for Medicaid and Medicare transformed

13 payment structures with a focus on hospital readmissions, and national physicians organizations

14 developed a Transitions of Care Consensus Policy Statement. ${ }^{5-7}$ Accordingly, the phrase

15 "transition of care" is widely understood to describe the changes in setting, healthcare providers,

16 and disease management strategies experienced by hospitalized patients at the time of discharge.

17 Like hospital discharge, hospital admission involves transitions in sites of care, handoffs between

18 healthcare providers, and changes in medical therapies. Both are associated with significant

19 stress to patients and their families. ${ }^{8,9}$ While hospital discharge processes have been the focus of

20 tremendous research, policy, and quality improvement efforts, research determining quality of

21 care at the time of hospital admission is scant. ${ }^{5,9,10}$ As a result, hospital admissions expose

22 patients to many of the same risks that have been the focus of hospital discharge reform:

23 unstructured patient handoffs, poor communication between healthcare providers, and inefficient 
1

2

3 In the US, patients in need of hospital admission enter the hospital through one of two primary

4 mechanisms: via the emergency department (ED) or via direct admission, defined as admission

5 to hospital from the community without first receiving care in the hospital's ED. Over the last

6 two decades EDs have increasingly served as portals for hospital admission, contributing to care

7 fragmentation and ED crowding. ${ }^{11}$ In a landmark report, the Institute of Medicine describes our

8 emergency medical system as overburdened, fragmented, and at the breaking point. ${ }^{12}$ Yet, of the

$9 \quad 1.5$ million non-elective pediatric hospitalizations that occur each year, $75 \%$ originate in EDs

10 with the remainder occurring via direct admission. ${ }^{13}$

11

12 While ED utilization patterns have been well studied, there is a paucity of research comparing

13 the effectiveness of direct and ED admissions, particularly in children. ${ }^{11,14,15}$ Direct admission

14 may offer benefits for both patients and healthcare systems, including reduced ED volumes,

15 improved coordination between outpatient and hospital-based healthcare providers, and

16 improved family experience of care. Currently, direct admission rates vary substantially across

17 hospitals and conditions, with condition-specific direct admission rates for unplanned

18 hospitalizations ranging from $9 \%$ for appendectomy to $38 \%$ for bipolar disorder. ${ }^{13}$ These

19 quantitative data are mirrored in a national survey of pediatric medical directors reporting direct

20 admission rates at their hospitals that range from less than $10 \%$ to greater than $50 \% .{ }^{16}$ Despite

21 this, only one-third report having formal direct admission policies in place, while 50\% report a

22 belief that more children should be admitted directly. This significant variation across hospitals

23 and conditions indicates clinical uncertainty regarding hospital admission best practices. We 
1 therefore propose a multi-site stepped wedge randomized controlled trial to: (i) compare the

2 effectiveness of direct admission to admission through the ED, and (ii) evaluate the causal effect

3 of implementing a direct admission program.

4

5 Methods

6 Aims

7 The overall goal of this project is to compare the effectiveness of a standardized direct admission

8 approach to admission beginning in the ED for hospitalized children. We will achieve this goal

9 by addressing the following specific aims: (1) Determine the effect of a pediatric direct

10 admission intervention on timeliness of healthcare provision, family experience of care, and rates

11 of clinical deterioration compared to pediatric hospital admission beginning in the ED; (2)

12 Identify the pediatric populations and conditions that experience the greatest benefits from direct

13 admission with respect to timeliness of healthcare provision and family experiences of care; and

14 (3) Identify barriers to and facilitators of implementing direct admission processes by applying

15 qualitative and quantitative methods.

16

17 For Aim 1, we hypothesize that a direct admission will be associated with more rapid initiation

18 of clinical care and improved family experience of care with no significant differences in rates of

19 clinical deterioration compared with admission beginning in the ED. Further, we hypothesize

20 that by making direct admission to hospital from a primary or urgent care practice available, we

21 will reduce the average time until initiation of clinical care across all patients (whether admitted

22 directed or via the ED). For Aim 2, we hypothesize that children with urinary tract infections

23 (UTIs) or skin and soft tissue infections (SSTIs) will experience the greatest benefits from direct 
1 admission relative to other conditions examined, as these conditions generally require minimal

2 diagnostic testing or specialized equipment prior to treatment initiation. We further hypothesize

3 that children with complex chronic conditions will experience greater benefits than children

4 without these conditions, as they are often well known to the hospital-based healthcare teams and

5 may be more likely to receive timely, personalized care. Underlying these hypotheses is our

6 theory that, by eliminating ED wait times and exposure to the often hectic ED environment, and

7 by reducing the number of healthcare teams involved in care provision, directly admitted

8 children will have reduced time to clinical care and improved experiences.

Theoretical Framework

11 Direct admission to hospital has several prerequisites, including access to timely ambulatory care

12 for children with acute illnesses, and communication between outpatient and inpatient healthcare

13 providers to initiate direct admission referrals. Our conceptual framework is modified from

14 Donabedian's structure, process, outcome framework to also incorporate patient characteristics

15 and family preferences (Figure 1). ${ }^{17}$ This framework illustrates how systems factors and

16 processes of care may influence where children access ambulatory care for acute illnesses (ED,

17 urgent care centers, or primary care clinics) and whether admission occurs directly or through the

18 ED. These factors, in turn, influence health outcomes. The system factors summarized in this

19 model, including clinic and ED factors, were described by parents of hospitalized children as

20 influential to their decisions about when and where they seek care for their child's acute

21 illnesses. ${ }^{8}$ The processes of care factors are derived from deliberative discussions with

22 stakeholders in the hospital admission process. ${ }^{18}$

23 Design 
1 We will conduct a cluster randomized controlled trial (RCT) at 3 structurally and geographically

2 diverse hospitals to compare the effectiveness of direct admission to admission through the ED,

3 and to evaluate the causal effect of implementing a direct admission program. To perform theses

4 analyses we will randomize 70 primary and urgent care practice sites in the hospitals' catchment

5 area to a time point for crossing over to the direct admission intervention (Figure 2); leadership

6 at each site will agree to randomization prior to project start. This crossover will be

7 unidirectional and occur at 4 time points, six months apart, over the 24-month implementation

8 period. Practices in each group will be randomized using a stratified approach, with 17-18

9 practice sites (4-9 practice sites per hospital) randomized to crossover to the direct admission

10 intervention at each step/crossover point, stratified by hospital and practice group, with the

11 random allocation conducted by AJO. In preparing this protocol report, we followed

12 Consolidated Standards of Reporting Trials extension for Cluster Trials (Additional File 1). ${ }^{19}$

$14 \quad$ Setting

15 The study will be carried out at three health systems in the US, selected for their structural 16 diversity, current low direct admission rates, and strong institutional support to implement and

17 evaluate direct admission processes. The first system has a freestanding children's hospital with

18 a pediatric ED annual volume of over 80,000 visits and a pediatric hospital medicine (PHM)

19 service that provides care to more than 9000 children each year. At this site, four ambulatory

20 care groups will participate in the intervention including one practice group owned by the

21 hospital system and three practice groups affiliated with the hospital through a quality of care

22 alliance. Across these four ambulatory care groups, 34 unique primary and urgent care practice

23 sites will be randomized to participate in this study. The second participating health system also 
1 has a freestanding children's hospital with a pediatric ED annual volume greater than 90,000

2 visits, a PHM service that provides care to 6000 children each year, and a pediatric infectious

3 disease service that admits approximately 3400 hospitalizations yearly. At this hospital, there are

418 ambulatory care partners, including 12 primary care practices and 6 urgent care centers, all of

5 which are owned by the health system. Our third system has a general hospital where children

6 seeking care in their ED are cared for in a general (non-children's) ED by general (non-pediatric

7 fellowship-trained) emergency medicine physicians. This hospital admits approximately 650

8 children annually and the ambulatory care partners at this site include three primary care groups

9 who practice across 18 unique primary care sites and affiliated urgent care centers.

11 Comparators \& Study Eligibility

12 At the patient level, we will evaluate the comparative effectiveness of direct versus ED

13 admission. At the system level, we will compare outcomes in children who are admitted from

14 eligible primary and urgent care practices where the direct admission intervention is available

15 (including children admitted directly and through EDs) to those admitted from practices not yet

16 eligible for the direct admissions intervention (only ED admission is available). Eligible children

17 will be nested within their respective primary care practices. We will limit our analysis to

18 children $<18$ years of age admitted with the following common medical reasons for

19 hospitalization: gastroenteritis, dehydration, SSTI, UTI/pyelonephritis, pneumonia, influenza,

20 and viral infection not otherwise specified. These diagnoses comprise approximately $25 \%$ of all

21 unscheduled pediatric admissions nationally and represent conditions that have been identified as

22 appropriate for direct admission but not currently accepted for direct admission at the

23 participating hospitals. ${ }^{1,18}$ Children with planned admissions (e.g. chemotherapy), those admitted 
1 to the intensive care unit or other subspecialty services, and those transferred from other

2 hospitals will be excluded.

3 Intervention Core Elements

4 The direct admission intervention will involve five core elements, endorsed by a national multi-

5 stakeholder panel: ${ }^{18}$ (i) direct admission education and tools for both referring healthcare

6 providers (e.g., primary care physicians (PCPs)) and accepting healthcare providers (nurses,

7 resident physicians and pediatric hospitalists), including diagnoses and populations eligible for

8 direct admission, and patient referral methods; (ii) a system to facilitate direct communication

9 between referring and accepting healthcare providers, enabling a single telephone call from a

10 referring physician to an accepting hospital-based physician (currently in place at all hospitals to

11 facilitate acceptance of inter-hospital transfers), and use of a structured data collection tool to

12 facilitate determination of the appropriateness of the patient for direct admission; (iii)

13 instructions for families regarding when/where/how to proceed for direct admission; (iv) rapid

14 evaluation of clinical stability of the patient upon hospital arrival by the inpatient healthcare

15 team; and (v) timely initiation of appropriate clinical care.

17 Local Adaptation and Leadership Teams

18 Each site will develop a multi-stakeholder Direct Admissions Leadership Team (DALT),

19 comprised of key stakeholders in the hospital admission process including pediatric hospitalists,

20 nurses, patient and family representatives, PCP representatives, and hospital administrators.

21 During the pre-implementation period, each DALT will meet to discuss intervention elements

22 within the context of local system, organization, provider, and patient characteristics. These

23 discussions will be supported by review of local administrative data and pilot data of study 
1 outcomes, with tailoring of educational materials and tools as appropriate. During the

2 implementation phase, the DALT and central research team will meet to discuss implementation

3 fidelity and the need for potential adaptation.

4

5 Outcome measures and data collection

6 Our primary outcome is timeliness of clinical care provision, defined as the time from arrival at

7 the hospital until initiation of clinical care by the accepting healthcare provider (including

8 diagnostic testing and/or medical management). This outcome will be derived from electronic

9 health record (EHR) time stamps; all project sites use EHRs to document times of patient

10 registration, orders, administration of medications and therapies, and diagnostic tests. EHR time

11 stamps have been previously validated using time motion data $;{ }^{20}$ during the pre-implementation

12 period we will similarly validate the accuracy of time stamps at each hospital.

14 Secondary outcomes include: (i) parent-reported family experience of care; (ii) unanticipated

15 transfer to the intensive care unit (ICU) within 6 hours of hospital admission, a marker of clinical

16 deterioration; ${ }^{21}$ and (iii) rapid response calls within 6 hours of hospital admission, a second

17 marker of clinical deterioration defined as calls to the hospital's medical-emergency team placed

18 by any person concerned about signs of critical clinical deterioration.

20 Family experience of care will be determined by parent-report using an instrument modified

21 from existing experience of care measures, including the Child Hospital Consumer Assessment

22 of Healthcare Providers and Systems Survey (Child HCAHPS ${ }^{22}$ and the Consumer Quality

23 Index. ${ }^{23}$ Following instrument development and pilot testing, this parent-reported outcome will 
1 be administered via electronic tablet to parents/guardians between six and seventy-two hours

2 following hospital admission.

3

$4 \quad$ Predictor Variables

5 We will evaluate, at baseline, patient level characteristics that may be associated with portal of

6 hospital admission. These include: child age, gender, race/ethnicity, primary payer (Medicaid,

7 commercial, other), primary reason for hospital admission (clinical condition), medical

8 complexity, and level of care (observation or inpatient status). Medical complexity will be

9 categorized as no complex chronic conditions, one complex chronic condition (i.e. respiratory

10 disease), or two or more complex chronic conditions (i.e. respiratory and neuromuscular disease)

11 by applying a previously established algorithm. ${ }^{24}$

12

13 Analytic Plan

14 For Aim 1, we will conduct two analyses. The first analysis will compare the effectiveness of

15 direct and ED admission at the patient level using an observational approach. The second

16 analysis will evaluate our hypothesis that the availability of a direct admission program will be

17 associated with an overall increase in the rapidity of initiation of clinical care and improved

18 family experience of care with no significant differences in rates of clinical deterioration

19 compared to not having direct admission available. In this second analysis, due to randomization,

20 we expect the distributions of patient characteristics to be balanced between practices eligible for

21 direct admission and those not yet randomized to the direct admission program. However, the

22 cluster-randomized structure of the study allows for the possibility that non-trivial imbalances in

23 patient covariates could occur between the study arms. Therefore, prior to analyzing any 
1

2

3

4

outcomes, we will compare the distribution of baseline covariates between the groups. We will then examine unadjusted differences in our outcomes. To adjust for observed predictors related to direct and ED admission, including temporal trends, for our main analyses we will use regression models. Because all patients receive some form of clinical care at the hospital irrespective of the form of admission, there is no censoring associated with the measurement of time to clinical care. As a result, standard regression models may be used.

\section{Analysis I: comparative effectiveness of direct and ED admission}

The statistical model for our patient level comparison of direct admission versus ED admission is an observational analysis due to patients not being randomly assigned to admission type. This model (i) is represented as:

$$
Y_{i j h t}=\beta_{0}+\beta_{1} D A_{i j h t}+\beta_{2} x_{i j h t}+\beta_{3 t}+\beta_{4 h}+\theta_{j h}+\varepsilon_{i j h t}
$$

where $Y_{i j h t}$ is the outcome variable and $D A_{i j h t}$ is a binary variable indicating whether the patient was admitted via direct admission $\left(D A_{i j h t}=1\right)$ or via the ER $\left(D A_{i j h t}=0\right)$ for patient $i$ seen by clinic $j$ at hospital $h$ at time $t$. In addition, $x_{i j h t}$ is a vector of patient level controls and $\theta_{j h}$ is a random effect for clinic within hospital. The model also includes fixed-effects for time-period, $\beta_{3 t}$, and hospital, $\beta_{4 h}$, to capture the general unstructured trend across calendar time and hospital-specific effects, respectively. The key coefficient of interest is $\beta_{1}$, which captures the association of a patient being admitted directly versus via the ED. In order to avoid contamination from outcomes of ED observations when direct admission was not available, for this analysis we plan to only use observations from the post-periods. However, to gain some insight into the closeness of the results from this analysis with those that we'd expect to see if it was possible to have patient level randomization, in a supplemental analysis we will include the pre-intervention ED observations and test if there is a change in the mean ED outcome in the 
1 post-intervention period. A finding of minimal change over time will support a hypothesis that,

2 in the absence of the intervention, the outcomes for direct admission patients would have been

3 the same as those observed for patients admitted through the ED.

$5 \quad$ II. Causal Analysis

6 In addition to the above-described patient level analysis, we will analyze the data under the

7 cluster-randomized stepped wedge design to estimate the causal effect of the availability of

8 direct admission on our primary outcome, time to receipt of clinical care. The key predictor in

9 this analysis is a clinic-level time-varying predictor indicating whether or not direct admission is

10 available, with its coefficient capturing the effect of direct admission availability on the average

11 time until clinical care is received across all patients, whether or not they were directly admitted.

12 The statistical model for this analysis (ii) has the form:

$$
Y_{i j h t}=\beta_{0}+\beta_{1} \text { Post }_{j h t}+\beta_{2} x_{i j h t}+\beta_{3 t}+\beta_{4 h}+\theta_{j h}+\varepsilon_{i j h t}
$$

14 in which $Y_{i j h t}$ is the outcome variable for patient $i$ seen by clinic $j$ at hospital $h$ at time $t$ while

15 Post $_{j h t}$ is a time-varying practice within hospital indicator of whether the intervention had been

16 rolled-out in clinic $j$ at hospital $h$ by time $t(1=$ intervention transition has occurred by time

17 period $t$ and $0=$ direct admission intervention has not occurred by time-period $t$ ) at the time

18 when the observation is made. The other predictors are as defined for model (i). The key

19 coefficient of interest is $\beta_{1}$, which captures the structural shift in the outcome that occurs when a

20 practice receives the direct admission intervention. Because Post $_{j h t}$ does not vary within a clinic

21 and time-period, the information in the observations made within a clinic and time-period

22 depends on the size of the effects of clinic and time-period and the statistical significance of 
1 inference about $\beta_{1}$ is likely to be reduced by the clustering of observations in clinics. An

2 additional component of clustering arises because the four groups are aligned with nine practice

3 groups that in turn are embedded in three hospitals; clustering is of greater concern for this

4 analysis as the key predictor only varies by cluster-time units, not among individuals within

5 cluster-time units. We acknowledge that there may be separate levels of clustering in the data

6 due to hospital and practice groups. However, the nine practice groups emanating from the 3

7 hospitals will be randomly distributed across the four groups of the stepped wedge design in a

8 manner that balances the four groups with respect to the characteristics of these groups. Because

9 the practice groups and hospitals are cross-classified with the four groups in our stepped wedge

10 design, their effects on the statistical precision of the results will be much less than under a

11 purely hierarchical arrangement.

13 For Aim 2, to test our hypothesis that children with UTI, SSTI, and complex chronic conditions

14 will experience the greatest benefits from direct admission relative to children admitted with

15 other clinical diagnoses, we will evaluate group-level heterogeneity of treatment effects (HTE)

16 by conducting subgroup analyses as specified a priori. Although there is no prior literature about

17 the relative benefits of direct admission for these subgroups, this hypothesis is derived from our

18 deliberative discussions with multidisciplinary stakeholders, based on their lived experiences.

19 Our anticipated sample size for subgroups is derived from national statistics about pediatric

20 inpatient stays, shown in Table 1. Although our assessment of HTE will follow methodological

21 guidance that all subgroups be specified a priori, we will consider other subgroup analyses based

22 on recommendations from Parent Partners and hospitals' DALTs. ${ }^{25,26}$ Subgroup analyses will be

23 performed by estimating an analogous model to that for the above-described patient level 
1 analysis. To evaluate whether the effect of the direction admission intervention varies

2 significantly across subgroups, we will augment the model specified in the Aim 1 analysis with a

3 predictor for the product of the subgroup-defining variable and direct admission; the coefficient

4 of the resulting variable is referred to as an interaction effect and its value captures the extent to

5 which the effect of direct admission differs between one level of the subgroup to the other. This

6 approach is based on the recommendations by Kent et al., who recommend analysis and

7 reporting of multivariate risk-based HTE to account for the fact that patients have multiple

8 characteristics simultaneously that affect the likelihood of benefiting from an intervention. ${ }^{26,27}$

10 Lastly, for Aim 3, in order to determine the impact of our direct admission intervention and to

11 inform post-project implementation in other health systems, we will conduct a mixed-methods

12 process evaluation applying the RE-AIM implementation framework to assess reach,

13 effectiveness, adoption, implementation and maintenance of our direct admission intervention. ${ }^{28}$

14 These domains, definitions, and our proposed approach are summarized in Table 2. This

15 approach combines analysis of our primary and secondary outcomes with analysis of process measures and qualitative interviews with stakeholders. Semi-structured interviews focused on the

17 adoption, implementation, and maintenance domains will be conducted with parents of hospitalized children, referring PCPs, and inpatient healthcare team members including nurses,

19 resident physicians, hospitalists, and other key stakeholders. We anticipate completing 12-16 in-

20 depth interviews with stakeholders at each hospital system each year, for a total of 72-96

21 interviews over the 2-year implementation period. However, interviews will be continued until

22 thematic saturation is reached. ${ }^{29}$ Interviews will be recorded with permission, transcribed

23 verbatim, and analyzed iteratively using a general inductive approach. ${ }^{30-32}$ 


\section{Sample Size and Statistical Power}

2 Based on review of administrative data from participating sites, we anticipate that 190 children and adolescents will be directly admitted, with 1506 admitted through the ED over the 24-month implementation period (Figure 2). We expect a 100\% response rate for our primary outcome, which will be derived from EHR data available for all hospitalized children by hospital protocol and procedures. This is also true of our secondary outcomes derived from EHR data, including ICU transfer and rapid response calls. However, for our parent-reported measure of family experience of care, we estimate a response rate of $75 \%$, accounting for potential parental refusals, absence of parents from the bedside, and inability to complete the survey in English, Spanish, Arabic, Nepali, or Somali. ${ }^{33,34}$ Of those admitted through EDs, we estimate that 870 will be during post time-periods. Therefore, for the patient level analysis described above, we anticipate 190 direct admissions and 870 concurrent control ED admissions.
Because the effects of all forms of clustering in the patient level observational analysis of Aim 1 are minimal, we illustrate the statistical power for this analysis by using a two-group t-test. To inform our power calculations, we collected pilot data from a pediatric hospital medicine program with a well-established direct admission program, applying similar eligibility criteria and definitions proposed for this study. In this pilot study, we found that mean time to clinical care for ED admissions was 85 min [SD $83 \mathrm{~min}$ ], and mean time for direct admissions was 40 minutes [SD $28 \mathrm{~min}]$.
With the above means and standard deviations, a two-sided 0.05-level test that allows for unequal standard deviations performed in the context of the patient level observational analysis 
1 of Aim 1 has power greater than 0.99 . There is also substantial power for subgroup analyses. For

2 example, the power of a subgroup analysis that involves $10 \%$ of the sample (applied evenly

3 across clinics and time-intervals) has power of 0.98. It is only when the subgroup is as small as

$45 \%$ that power falls below 0.80 . This power calculation was performed using Satterwaite's

5 approximation (i.e., evaluating a pooled variance to approximate the sampling distribution of the

6 actual test-statistic by a t-distribution) under nQuery. ${ }^{35,36}$

7

8 For the system-level stepped wedge analysis, we compute power for the effect of the predictor

9 labelled Post, the effect of a clinic receiving the intervention and entering the post-period, for the analysis of the entire sample. The method of computing power for a design with unequal

11 standard deviations in the prior and post time-periods is to first determine the design-effect for

12 the stepped-wedge design and then compute the power for a two-population comparison using

13 the effective-sample-sizes based on the design-effect. The design-effect is estimated using the 14 expression in Woertman et al (2013), also described in Hemming (2016). ${ }^{37,38}$ Because hospital and practice group are cross-classified across the four stepped wedge design groups, and because

16 little is known about likely levels of interclass correlation coefficients (ICC) for each of hospital,

17 practice group, and clinic, we perform illustrative power calculations assuming that the net

18 impact of clustering is equivalent to hospital-level clustering alone with an ICC of hospital of

19 0.05. Based on results for past cluster-randomized studies at the clinic level, an intraclass

20 correlation of 0.05 appears to be a conservative estimate of clustering by clinic. ${ }^{39,40}$ It is not

21 unreasonable for 0.05 to still be conservative once the upper-level sources of cross-classified

22 clustering are absorbed. The other inputs are 70 practice sites (clusters), 4 waves of intervention

23 onset, no-baseline period, 1,696 patients in total and equal numbers of patients per clinic per 
1 time-interval. Under this scenario the design-effect is 2.385. Therefore, the effective sample-size

2 (ESS) is 711.

4 The second part of the calculation is to determine the power of a two-group comparison of a

5 continuous outcome in the absence of clustering when the total sample-size is 711 . Because there

6 are 10 group times in the post-period and 6 in the pre-period each with 106 observations, the split

7 in the ESS between the post and the pre-period is 444 and 267. The mean and standard deviation

8 of the outcome in the pre-period is given by the above values for ED admissions; the mean is 85

9 minutes and the standard deviation is 83. To determine the mean and standard deviation of the

10 outcome expected in the post-period, we take weighted averages of the direct admission and ED

11 admission means and standard deviations using the total theorem of probability to compute

12 marginal effects. The weight of direct admission to the total patients is $19 / 106=0.094$. The

13 weighted average marginal mean and weighted average marginal standard deviation of the

14 observations in the post-period are 76.93 and 84.99, respectively. Because the sample-sizes are

15 reasonably large, an asymptotic normal approximation is well justified (i.e., even if the true

16 distribution of the outcome is bimodal it will be averaged out and converging towards

17 normality). Given the ESS's and these means and standard deviations, a two-sided 0.05-level test

18 has power of 0.23 . In order to have 0.80 power, we would need a much larger effect size.

19 Specifically, the mean for the post-period would need to be approximately 66.5. Due to the small

20 fraction of patients who are admitted directly, this will only be plausible if there is a spillover

21 effect of the intervention such that the outcome is reduced for both patients admitted directly and 22 via the ED. 
1

2 With the exception of our parent-reported outcome, all required data for this project will be

\section{Data management}

4

列

extracted from the hospitals' EHRs; all proposed variables exist in EHRs, thereby minimizing data collection burden and missingness as a result of non-response. During the preimplementation period, all sites will conduct pilot data extraction from EHRs to assess data source adequacy; any deficiencies will be addressed prior to the rollout. Following data extraction at hospital sites, de-identified data will be sent via HIPAA-secure methods to the research hub, where all data analyses will be conducted and subsequently shared with sites.

\section{Discussion}

Our study represents the first RCT to compare the effectiveness of direct and ED admission for children. We will implement a multi-stakeholder informed admission process and evaluate outcomes across diverse settings to advance our understanding of factors that support and hinder an effective direct admission process. Our scientific approach, pairing a robust design with a multi-level assessment of barriers to and facilitators of implementation, will generate valuable data to inform implementation of direct admission programs at other healthcare systems nationally.

A better understanding of the comparative effectiveness of direct versus ED admission is important to parents of hospitalized children and to their healthcare teams. Parents and physicians recognize potential benefits and challenges of direct and ED admission and articulate a desire for standardized admission processes ${ }^{8,16}$ Consistent with the Institute of Medicine's domains of healthcare quality, parents describe the importance of timely, effective, safe, and 
1 patient-centered clinical care, but express variation in their experiences with direct and ED

2 admissions in achieving these aims. ${ }^{8}$ In addition, the growth of pediatric hospital medicine in the

3 US, the pediatric specialty dedicated to the inpatient care of children, creates both opportunities

4 for around-the-clock pediatric-specific care but also creates a discontinuity of care between

5 outpatient and inpatient providers. ${ }^{41-43}$ Implementation of effective direct admission programs

6 has the potential to improve patient hand-offs, communication between healthcare providers,

7 efficiency and timeliness of care, and patient and family experience.

\section{Dissemination}

We anticipate several peer-reviewed manuscripts and presentations resulting from this work.

Beyond this, we will disseminate our findings to our health system partners and via national

12 organizations, social media, press releases, and media alerts. ${ }^{33}$

\section{Declarations:}

Ethics: IRB approval was obtained through the Dartmouth College Committee for the Protection of Human Subjects (\#31814) with implementation sites ceding review to Dartmouth.

Consent for publication: not applicable

Availability of data and materials: Not applicable as no datasets were generated or analyzed for the production of this study protocol.

Competing interests: The authors declare that they have no competing interests.

Funding: This work was supported through a Patient-Centered Outcomes Research Institute (PCORI) Project Program Award (IHS-2018C2-12902-IC).

Disclaimer: All statements in this report, including its findings and conclusions, are solely those of the authors and do not necessarily represent the views of the Patient-Centered Outcomes 
1

2 Authors contributions: All authors made substantial contributions to the conception, design, 3 analysis or interpretation of this work. JL, CM, and JAO drafted the manuscript, and MB, AS 4 and SA critically reviewed or revised it. All authors have approved the submitted work. 


\section{REFERENCES}

1. Leyenaar JK, Ralston SL, Shieh M, Pekow PS, Mangione-Smith R, Lindenauer PK. Epidemiology of Pediatric Hospitalizations at General Hospitals and Freestanding Children's Hospitals in the United States. J Hosp Med. 2016;0(0):1-7. doi: $10.1002 / \mathrm{jhm} .2624$.

2. Lassman D, Hartman M, Washington B, Andrews K, Catlin A. US health spending trends by age and gender: selected years 2002-10. Health Aff (Millwood). 2014;33(5):815-822. doi:10.1377/hlthaff.2013.1224.

3. Moore B, Levit K, Elixhauser A. Ph.D.Costs for Hospital Stays in the United States, 2012. Healthcare Cost and Utilization Project 181. Available at: http://www.hcupus.ahrq.gov/reports/statbriefs/sb181-Hospital-Costs-United-States-2012.pdf. Accessed Septe.

4. Thomson J, Shah SS, Simmons JM, et al. Financial and Social Hardships in Families of Children with Medical Complexity. J Pediatr. 2016;172:187-193.e1. doi:10.1016/j.jpeds.2016.01.049.

5. Snow V, Beck D, Budnitz T, et al. Transitions of Care Consensus Policy Statement American College of Physicians-Society of General Internal Medicine-Society of Hospital Medicine-American Geriatrics Society-American College of Emergency PhysiciansSociety of Academic Emergency Medicine. J Gen Intern Med. 2009;24(8):971-976. doi:10.1007/s11606-009-0969-x.

6. Jack BW, Chetty VK, Anthony D, Greenwald JL, Sanchez GM. A Reengineered Hospital Discharge Program to Decrease Rehospitalization: a randomized trial. Ann Intern Med. 2009;150:178-187.

7. Kangovi S, Grande D. Hospital readmissions--not just a measure of quality. JAMA. 2011;306(16):1796-1797. doi:10.1001/jama.2011.1562.

8. Leyenaar J, Pizzo P, O’Brien E, Lindenauer P. Paediatric hospital admission processes and outcomes: a qualitative study of parents' experiences and priorities. BMJ Qual Saf. 2018;Epub ahead.

9. Leyenaar J, O’Brien E, Leslie L, Lindenauer P, Mangione-Smith R. Families' priorities regarding hospital-to-home transitions for children with medical complexity. Pediatrics. 2017;139(1):pii: e20161581.

10. Coleman E a. Safety in numbers: physicians joining forces to seal the cracks during transitions. J Hosp Med. 2009;4(6):329-330. doi:10.1002/jhm.548.

11. Tang N, Stein J, Hsia RY, Maselli JH, Gonzales R. Trends and characteristics of US emergency department visits, 1997-2007. JAMA. 2010;304(6):664-670. doi:10.1001/jama.2010.1112.

12. Institute of Medicine. Hospital-based emergency care: At the breaking point. Washington, DC: National Academies Press. 2006.

13. Leyenaar JK, Shieh M-S, Lagu T, Pekow PS, Lindenauer P. Direct Admission to Hospitals Among Children in the United States. JAMA Pediatr. 2015;169(5):500-502.

14. Schuur J, Venkatesh A. The growing role of emergency departments in hospital admissions. N Engl J Med. 2012;367(5):391-393. doi:10.1056/NEJMp1206519.

15. Bourgeois FT, Monuteaux MC, Stack AM, Neuman MI. Variation in Emergency Department Admission Rates in US Children's Hospitals. Pediatrics. 2014;134(3):539545. doi:10.1542/peds.2014-1278. 
16. Leyenaar JK, O'Brien ER, Malkani N, Lagu T, Lindenauer PK. Direct Admission to Hospital: A Mixed Methods Survey of Pediatric Practices, Benefits, and Challenges. Acad Pediatr. August 2015:1-8. doi:10.1016/j.acap.2015.07.002.

17. Donabedian A. Evaluating the quality of medical care. Milbank Mem Fund $Q$. 1966;44(3):166S-206S.

18. Leyenaar JK, Rizzo P, Shevenell M, Hill V, Lindenauer P. Multi-stakeholder informed guidelines for pediatric direct admission to hospital. J Pediatr.

19. Campbell MK, Piaggio G, Elbourne DR, Altman DG, CONSORT Group. Consort 2010 statement: extension to the cluster randomized trials. BMJ. 2012;345:e5661.

20. Arndt B, Beasley J, Watkinson M, et al. Tethered to the EHR: Primary Care Physician Workload Assessment Using EHR Event Log Data and Time-Motion Observations. Ann Fam Med. 2017;15:419-427.

21. Reese J, Deakyne S, Blanchard A, Bajaj L. Rate of preventable early unplanned intensive care unit transfer for direct admissions and emergency department admissions. Hosp Pediatr. 2015;5(1):27-34.

22. Toomey SL, Zaslavsky AM, Elliott MN, et al. The Development of a Pediatric Inpatient Experience of Care Measure: Child HCAHPS ${ }^{\circ}$. Pediatrics. 2015;136(2):360-369. doi:10.1542/peds.2015-0966.

23. Smirnova A, Lombarts KMJMH, Arah OA, Vleuten CPM Van Der. Closing the patient experience chasm : A two- - level validation of the Consumer Quality Index Inpatient Hospital Care. Heal Expect. 2017;20:1041-1048. doi:10.1111/hex.12545.

24. Feudtner C, Feinstein JA, Zhong W, Hall M, Dai D. Pediatric complex chronic conditions classification system version 2: updated for ICD-10 and complex medical technology dependence and transplantation. BMC Pediatr. 2014;14(199):1-7.

25. Dahabreh IJ, Hayward R, Kent DM. Using group data to treat individuals : understanding heterogeneous treatment effects in the age of precision medicine and patient-centred evidence. Int J Epidemiol. 2016;45(6):2184-2193. doi:10.1093/ije/dyw125.

26. Kent DM, Rothwell PM, Ioannidis JPA, Altman DG, Hayward RA. Assessing and reporting heterogeneity in treatment effects in clinical trials : a proposal. Trials11. 2010;11(85):1-10.

27. Brookes ST, Whitely E, Egger M, Davey G, Mulheran PA, Peters TJ. Subgroup analyses in randomized trials : risks of subgroup-specific analyses ; power and sample size for the interaction test. J Clin Epidemiol. 2004;57:229-236. doi:10.1016/j.jclinepi.2003.08.009.

28. Glasgow RE, Vogt TM, Boles SM. Evaluating the Public Health Impact of Health Promotion Interventions : The RE-AIM Framework. Am J Public Health. 1999;89(9):1322-1327.

29. Guest G, Bunce A, Johnson L. How Many Interviews Are Enough?: An Experiment with Data Saturation and Variability. Field methods. 2006;18(1):59-82. doi:10.1177/1525822X05279903.

30. Creswell J, Miller D. Determining validity in qualitative inquiry. Theory Pract. 2000;39(3):124-130.

31. Creswell J. Qualitative Inquiry and Research Design: Choosing among Five Traditions. 3rd ed. Thousand Oaks, CA: Sage Publications Inc.; 2013.

32. Morse JM. Determining Sample Size. Qual Health Res. 2000;10(1):3-5.

33. Kascak L, Leyenaar JK. Sleep quality during pediatric hospital stays: a pilot study of patient experiences. Presented at Academic Pediatric Association Region 1 Meeting, 
Waltham MA, March, 2018.

34. Leyenaar JK, Shevenell M, Rizzo P, O’Neill M, Blaine K, Hussain, K, Mangione-Smith R, Berry JG. Hospital-to-home needs assessment for children with medical complexity: a pilot study of feasibility and acceptability. Pediatric Academic Societies Meeting, S.

35. Satterthwaite, FE. An Approximate Distribution of Estimates of Variance Components. Biometrics Bulletin, 1946: 2: 110-114, doi:10.2307/3002019.

36. nQuery. Available at: https://www.statsols.com/nquery/sample-size-software-options. Accessed July 7, 2020.

37. Hemming, K. Sample size calculations for stepped wedge trials using design effects are only approximate in some circumstances. Trials (2016) 17:234. DOI 10.1186/s13063-0161359-4.

38. Woertman W, de Hoop E, Moerbeek M, Zuidema SU, Gerritsen DL, Teerenstra S. Stepped wedge designs could reduce the required sample size in cluster randomized trials. J Clin Epidemiol. 2013;66(7):752-8. doi:10.1016/j.jclinepi.2013.01.009.

39. Kul S, Vanhaecht K, Panella M. Intraclass correlation coefficients for cluster randomized trials in care pathways and usual care: hospital treatment for heart failure. BMC Health Serv Res. 2014;14(84):1-7. doi:10.1186/1472-6963-14-84.

40. Thompson DM, Fernald D, Mold J. Intraclass Correlation Coefficients Typical of ClusterRandomized Studies : Estimates From the Robert Wood Johnson Prescription for Health Projects. Ann Fam Med. 2012;10:235-240. doi:10.1370/afm.1347.INTRODUCTION.

41. Gosdin C, Simmons J, Yau C, Sucharew H, Carlson D, Paciorkowski N. Survey of academic pediatric hospitalist programs in the US: organizational, administrative, and financial factors. J Hosp Med. 2013;8(6):285-291. doi:10.1002/jhm.2020.

42. Oshimura J, Sperring J, Bauer BD, Rauch D a. Inpatient staffing within pediatric residency programs: work hour restrictions and the evolving role of the pediatric hospitalist. J Hosp Med. 2012;7(4):299-303. doi:10.1002/jhm.952.

43. Wachter R, L. G. Zero to 50,000 - The 20th Anniversary of the Hospitalist. N Engl J Med. 2016;375(11):2016-2018. 
Table 1. Components of mixed methods process evaluation using RE-AIM Framework

\begin{tabular}{|l|l|}
\hline Domain and Definition & Approach \\
\hline $\begin{array}{l}\text { REACH: the \% and } \\
\text { characteristics of children } \\
\text { eligible for the direct admission } \\
\text { intervention who were admitted } \\
\text { via this approach }\end{array}$ & $\begin{array}{l}\text { Monthly reports to primary care practices reporting } \\
\text { the number and \% of eligible children admitted via } \\
\text { direct admission; quarterly reports displaying clinical } \\
\text { and sociodemographic characteristics of children } \\
\text { admitted directly compared to those admitted via the } \\
\text { ED }\end{array}$ \\
\hline $\begin{array}{l}\text { EFFICACY: consideration of } \\
\text { positive and negative outcomes } \\
\text { of the intervention }\end{array}$ & $\begin{array}{l}\text { Quarterly reports to primary care practices of primary } \\
\text { and secondary study outcomes }\end{array}$ \\
\hline $\begin{array}{l}\text { ADOPTION: barriers to and } \\
\text { facilitators of adopting this } \\
\text { intervention }\end{array}$ & $\begin{array}{l}\text { Qualitative interviews with key stakeholders will } \\
\text { focus on: (i) experience with the direct admission } \\
\text { intervention and (ii) barriers to and facilitators of a) } \\
\text { referral for direct admission, b) delivery of the } \\
\text { intervention, including adherence to core components } \\
\text { c) provision of timely and patient-centered care, and } \\
\text { d) assurance of patient safety }\end{array}$ \\
\hline $\begin{array}{l}\text { IMPLEMENTATION: the } \\
\text { extent to which the intervention } \\
\text { is delivered as intended }\end{array}$ & $\begin{array}{l}\text { Qualitative interviews with stakeholders, and } \\
\text { quarterly meetings of the Direct Admission } \\
\text { Leadership Teams to discuss barriers to and } \\
\text { facilitators of adherence to intervention }\end{array}$ \\
\hline $\begin{array}{l}\text { MAINTENANCE: the extent to } \\
\text { which the intervention is } \\
\text { sustained over time }\end{array}$ & $\begin{array}{l}\text { Monthly reports to primary care practices regarding } \\
\text { the number and \% of eligible children admitted via } \\
\text { direct admission, demonstrating changes over time }\end{array}$ \\
\hline
\end{tabular}


Table 2. Projected subgroup sample sizes

\begin{tabular}{|l|r|}
\hline $\begin{array}{l}\text { Condition / Population } \\
\text { (Total sample n=1696) }\end{array}$ & $\begin{array}{l}\text { Anticipated n } \\
(\boldsymbol{\%})\end{array}$ \\
\hline Medical Complexity: & $644(38 \%)$ \\
No chronic disease & $441(26 \%)$ \\
Chronic, non-complex & $611(36 \%)$ \\
Complex chronic disease & \\
\hline Diagnosis: & $551(33 \%)$ \\
Pneumonia & $262(16 \%)$ \\
Skin / soft tissue infection & $317(19 \%)$ \\
Gastroenteritis/dehydration & $182(11 \%)$ \\
Urinary Tract Infection & $293(18 \%)$ \\
Viral infection not & $51(3 \%)$ \\
otherwise specified & \\
Influenza & \\
\hline
\end{tabular}


Figure 1. Conceptual framework informing research approach

ACUTE AMBULATORY CARE: Clinic or ED

\begin{tabular}{|l|}
\hline \multicolumn{1}{|c|}{ SYSTEM FACTORS } \\
Clinic factors: \\
Hours of operation \\
Accessibility for acute care \\
EHR utilization \\
Clinic-hospital communication \\
infrastructure \\
ED factors: \\
Waiting environment \\
Availability of pediatric ED \\
providers \\
Communication infrastructure \\
Availability of diagnostic \\
testing \\
Access to specialists \\
\hline
\end{tabular}

PROCESSES OF CARE

Patient factors:

Diagnoses; disease severity

Co-morbidities/medical

complexity

Demographic characteristics

Family preferences

Healthcare delivery:

Direct admission policy and

procedures

Relationships: families, PCP,

ED and PHM providers

Wait times

Communicable disease

exposures

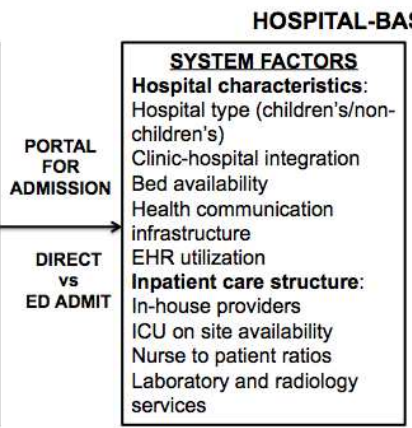

PROCESSES OF CARE

Patient factors:

Diagnoses; disease severity Co-morbidities/medical

Demographic characteristics

Family preferences

Healthcare delivery:

Communication with referring

provider

Triage, clinical evaluation

Hospital admission logistics

Order entry

Procedures \& investigations
HEALTH OUTCOMES

Timeliness of care

delivery

Patient and family

satisfaction/experience

of care

Clinical deterioration:

transfer to the ICU,

rapid response calls

* Donabedian A. Evaluating the quality of medical care. Milbank Mem Fund Q. 1966;44(3):166S-206S. 
Figure 2. Characteristics of stepped wedge cluster randomized controlled trial, where shaded areas indicate direct admission intervention exposure and unshaded areas indicated control conditions

TIME (0-36 month project period; each block represents 6 months)

\begin{tabular}{|c|c|c|c|c|c|c|}
\hline & $0 \mathrm{mo}$ & $6 \mathrm{mo}$ & $12 \mathrm{mo}$ & $18 \mathrm{mo}$ & $24 \mathrm{mo}$ & $30 \mathrm{mo}$ \\
\hline Group 1 & \multirow{8}{*}{$\begin{array}{l}\text { Pre-rollout } \\
\text { period }\end{array}$} & DA: 19 & DA: 19 & DA: 19 & DA: 19 & \multirow{8}{*}{$\begin{array}{l}\text { Post- } \\
\text { rollout } \\
\text { period }\end{array}$} \\
\hline (18 practices) & & ED: 87 & ED: 87 & ED: 87 & ED: 87 & \\
\hline Group 2 & & DA: 0 & DA: 19 & DA: 19 & DA: 19 & \\
\hline (18 practices) & & ED: 106 & ED: 87 & ED: 87 & ED: 87 & \\
\hline Group 3 & & DA: 0 & DA: 0 & DA: 19 & DA: 19 & \\
\hline (17 practices & & ED: 106 & ED: 106 & ED: 87 & ED: 87 & \\
\hline Group 4 & & DA: 0 & DA: 0 & DA: 0 & DA: 19 & \\
\hline (17 practices) & & ED: 106 & ED: 106 & ED: 106 & ED: 87 & \\
\hline
\end{tabular}

------------Rollout period--------------------------

$(\mathrm{DA}=$ direct admission; $\mathrm{ED}=\mathrm{ED}$ admission; numbers indicate projected sample 


\section{Figures}

ACUTE AMBULATORY CARE: Clinic or ED

\begin{tabular}{l}
\multicolumn{1}{c|}{ SYSTEM FACTORS } \\
Clinic factors: \\
Hours of operation \\
Accessibility for acute care \\
EHR utilization \\
Clinic-hospital communication \\
infrastructure \\
ED factors: \\
Waiting environment \\
Availability of pediatric ED \\
providers \\
Communication infrastructure \\
Availability of diagnostic \\
testing \\
Access to specialists \\
\hline
\end{tabular}

PROCESSES OF CARE Patient factors:

Diagnoses; disease severity

Co-morbidities/medical

complexity

Demographic characteristics

Family preferences

Healthcare delivery:

Direct admission policy and

procedures

Relationships: families, PCP,

ED and PHM providers

Wait times

Communicable disease

exposures

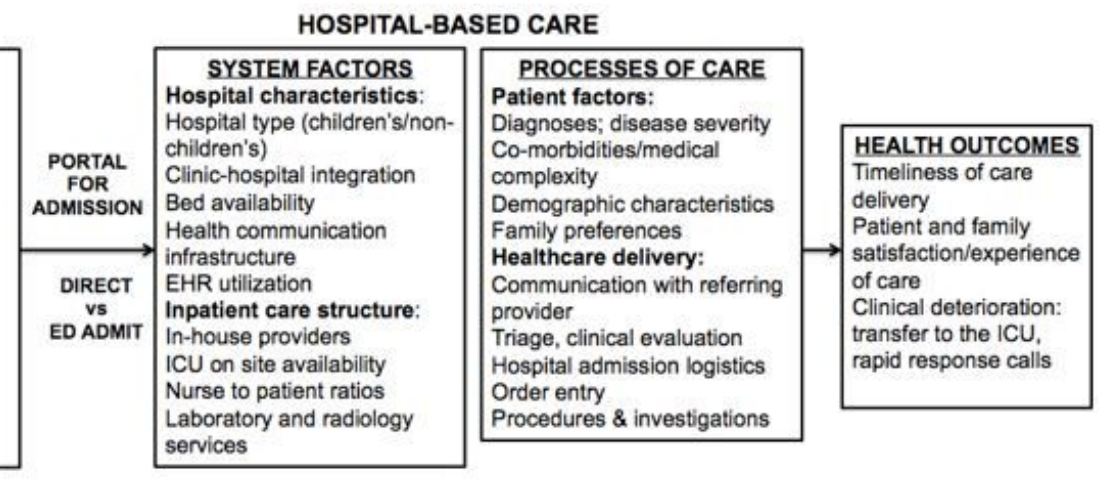

\section{Figure 1}

Conceptual framework informing research approach * Donabedian A. Evaluating the quality of medical care. Milbank Mem Fund Q. 1966;44(3):166S-206S.

TIME (0-36 month project period; each block represents 6 months)

\begin{tabular}{|c|c|c|c|c|c|c|}
\hline & $0 \mathrm{mo}$ & $6 \mathrm{mo}$ & $12 \mathrm{mo}$ & $18 \mathrm{mo}$ & $24 \mathrm{mo}$ & $30 \mathrm{mo}$ \\
\hline Group 1 & \multirow{8}{*}{$\begin{array}{l}\text { Pre-rollout } \\
\text { period }\end{array}$} & DA: 19 & DA: 19 & DA: 19 & DA: 19 & \multirow{8}{*}{$\begin{array}{l}\text { Post- } \\
\text { rollout } \\
\text { period }\end{array}$} \\
\hline (18 practices) & & ED: 87 & ED: 87 & ED: 87 & ED: 87 & \\
\hline Group 2 & & DA: 0 & DA: 19 & DA: 19 & DA: 19 & \\
\hline (18 practices) & & ED: 106 & ED: 87 & ED: 87 & ED: 87 & \\
\hline Group 3 & & DA: 0 & DA: 0 & DA: 19 & DA: 19 & \\
\hline (17 practices & & ED: 106 & ED: 106 & ED: 87 & ED: 87 & \\
\hline Group 4 & & DA: 0 & DA: 0 & DA: 0 & DA: 19 & \\
\hline (17 practices) & & ED: 106 & ED: 106 & ED: 106 & ED: 87 & \\
\hline
\end{tabular}

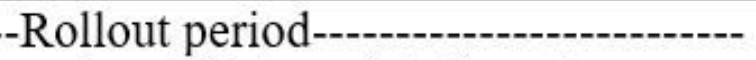

$(\mathrm{DA}=$ direct admission; $\mathrm{ED}=\mathrm{ED}$ admission; numbers indicate projected sample

Figure 2

Characteristics of stepped wedge cluster randomized controlled trial, where shaded areas indicate direct admission intervention exposure and unshaded areas indicated control conditions

\section{Supplementary Files}

This is a list of supplementary files associated with this preprint. Click to download. 
- ContractNo.IHS2018C212902IC.distIS.pdf

- CONSORTchecklist.docx

- CONSORTchecklist.docx 